\title{
Two-Dimensional Topology of the Sloan Digital Sky Survey
}

Fiona Hoyle ${ }^{1}$, Michael S. Vogeley ${ }^{1}$, J. Richard Gott III ${ }^{2}$, Michael Blanton ${ }^{3}$, Max Tegmark ${ }^{4}$, David H. Weinberg ${ }^{5}$, Neta A. Bahcall ${ }^{2}$, Jon Brinkmann ${ }^{6}$ \& Donald G. York ${ }^{7}$

1. Department of Physics, Drexel University, 3141 Chestnut Street, Philadelphia, PA 19104

2. Princeton University Observatory, Peyton Hall, Princeton, NJ 08544

3. Department of Physics, New York University, 4 Washington Place, New York, NY 10003

4. Department of Physics, University of Pennsylvania, Philadelphia, PA, 19101

5. Department of Astronomy, Ohio State University, 140 W. 18th Ave., Columbus, OH 43210

6. Apache Point Observatory, P. O. Box 59, Sunspot, NM 88349-0059

7. The University of Chicago, Astronomy \& Astrophysics Center, 5640 S. Ellis Ave., Chicago, IL, 60637

hoyle@venus.physics.drexel.edu, vogeley@drexel.edu, jrg@astro.princeton.edu

\begin{abstract}
We present the topology of a volume-limited sample of 11,884 galaxies, selected from an apparent-magnitude limited sample of over 100,000 galaxies observed as part of the Sloan Digital Sky Survey (SDSS). The data currently cover three main regions on the sky: one in the Galactic north and one in the south, both at zero degrees declination, and one area in the north at higher declination. Each of these areas covers a wide range of survey longitude but a narrow range of survey latitude, allowing the two dimensional genus to be measured.

The genus curves of the SDSS sub-samples are similar, after appropriately normalizing these measurements for the different areas. We sum the genus curves from the three areas to obtain the total genus curve of the SDSS. The total curve has a shape similar to the genus curve derived from mock catalogs drawn from the Hubble Volume $\Lambda$ CDM simulation and is similar to that of a Gaussian random field. Likewise, comparison with the genus of the 2dFGRS, after normalization for the difference in area, reveals remarkable similarity in the topology of these samples.

We test for the effects of galaxy type segregation by splitting the SDSS data into thirds, based on the $u^{*}-r^{*}$ colors of the galaxies, and measure the genus of the reddest and bluest sub-samples. This red/blue split in $u^{*}-r^{*}$ is essentially a split by morphology (Strateva et al. 2001). We find that the genus curve for the reddest galaxies exhibits a "meatball" shift of the topology - reflecting the concentration of red galaxies in high density regions - compared to the bluest galaxies and the full sample, in agreement with predictions from simulations.
\end{abstract}

Subject headings: cosmology: large-scale structure of the universe - cosmology: observations - galaxies: distances and redshifts - methods: statistical 


\section{Introduction}

Quantitative measurements of the large-scale structure of the galaxy distribution provide important constraints for models of structure formation. In particular, the topology of large-scale structure is a useful test of the initial conditions of the Universe. Inflation predicts that the seeds for structure formation should derive from a Gaussian random phase distribution (Bardeen, Steinhardt \& Turner 1983). The topology of the large-scale structure is invariant during the linear growth of structure. Thus, after appropriate smoothing, the topology of the present galaxy distribution can be related to that of the initial density field. The topology can be quantified using statistical methods, such as the genus statistic (Gott et al. 1986). This statistic allows a test of the random phase hypothesis, because any deviation of the measured topology on large smoothing scales might be evidence for non-Gaussian initial conditions. On relatively smaller scales, the topology of the smoothed galaxy density quantifies the degree of non-linear evolution and/or biasing of galaxy formation with respect to the mass density at the present epoch. The genus statistic quantifies the topology of the smoothed galaxy distribution by computing the integrated curvature of iso-density contours at different density thresholds. This statistic is complementary to statistical measures such as the power spectrum or correlation function, which quantify only the second moments of the distribution. By focusing on the connectedness of structure as a function of filling factor, the genus statistic also complements the 1-point probability distribution of smoothed density fields, which has a very different sensitivity to non-linearity, bias, and primordial non-Gaussianity. Analyses of early SDSS data include measurement of the power spectrum (Szalay et al. 2002; Tegmark et al. 2002; Dodelson et al. 2002), correlation function (Zehavi et al. 2002; Connolly et al. 2002; Infante et al. 2002) and higher order moments (Szapudi et al. 2002).

The genus statistic has been measured from a variety of galaxy surveys. Gott et al. (1989) applied the 3D genus statistic to small samples of galaxies. It has since been applied to subsequently larger surveys; the 3D genus of the SSRS was measured by Park, Gott \& da Costa (1992), Moore et al. (1992) applied it to the QDOT Survey, Rhoads et al. (1994) analyzed Abell Clusters, Vogeley et al. (1994) analyzed the CfA survey, Canavezes et al. (1998) analyzed the PSCz Survey, Protogeros \& Weinberg (1997) and Springel et al. (1998) analyzed the 1.2-Jy IRAS Survey. Hikage et al. (in preparation) estimate the 3D genus for an early sample of galaxies from the SDSS. Melott (1987) was the first to mention the possibility of studying the 2D topology, i.e. the genus of 1-dimensional contours of smoothed surface density in a thin slice redshift survey. This idea was further developed by Melott et al. (1989). The 2D genus method has been applied to the Lick Galaxy Catalog (Coles \& Plionis 1991), the Abell and ACO cluster catalogs (Plionis, Valdarnini \& Coles 1992), the CfA Survey (Park et al. 1992) and the LCRS survey (Colley 1997). Hoyle, Vogeley \& Gott (2002, HVG02) estimate the two-dimensional genus for the 2dF Galaxy Redshift. Some evidence was found for departure from Gaussianity that would be expected as a result of non-linear gravitational

evolution and/or biasing of galaxies as compared to the mass but on the whole, the results were consistent with the universe having Gaussian initial conditions.

In this paper, we estimate the genus of an initial sample of SDSS galaxies. This is the first time 
that a sample from the SDSS is large enough that measurements of the two-dimensional genus can be made. Because the SDSS includes not only medium-resolution spectroscopy but also five-band digital photometry for every object in the redshift sample, we can split the sample as a function of color, which is a good indicator of morphology. For the first time, we examine the two-dimensional genus of the reddest and bluest galaxies to see if genus is a function of galaxy type.

We describe the SDSS galaxy survey in Section 2 and summarize the method for measuring the genus in section 3 . We present our results in section 4 and we draw conclusions in section 5 .

\section{The Data Set and the Simulation}

\subsection{The Sloan Digital Sky Survey}

The SDSS is a wide-field photometric and spectroscopic survey. The completed survey will cover approximately 10,000 square degrees. CCD imaging of $10^{8}$ galaxies in five colors and followup spectroscopy of $10^{6}$ galaxies with $r^{\prime}<17.5$ will be obtained. York et al. (2000) provides an overview of the SDSS, Stoughton et al. (2002) describes the early data release (EDR) and details about the measurements. Technical articles providing details of the SDSS include descriptions of the photometric camera (Gunn 1998), photometric analysis (Lupton et al. 2002), the photometric system (Fukugita et al. 1996; Smith et al. 2002), the photometric monitor (Hogg et al. 2001), astrometric calibration (Pier et al. 2002), selection of the galaxy spectroscopic samples (Strauss et al. 2002; Eisenstein et al. 2001), and spectroscopic tiling (Blanton et al. 2001).

We examine a sub-sample that includes 63,125 galaxies with redshifts in the range $0.02<z<$ 0.20 and absolute magnitude limited to $-22.0<M_{r}<-19.0$. Some of these data have already been released to the community in the Early Data Release (see Stoughton et al. 2002). A thorough analysis of possible systematic uncertainties in the galaxy samples is described in Scranton et al. (2001). As we demonstrate, the sample is now large enough for meaningful two-dimensional genus measurements to be made.

To a good approximation, the sample we analyze consists of three great circle wedges, each approximately 5 degrees thick, with lengths of 70,100 , and 70 degrees. The physical thickness of these wedges is $8 h^{-1} \mathrm{Mpc}$ at the inner edge and $22 h^{-1} \mathrm{Mpc}$ at the outer edge. In detail, the three areas of interest are the stripe along the Celestial Equator in the Southern Galactic Cap (hereafter Area 1), which covers the region $350^{\circ}<\alpha<58^{\circ},-2.5^{\circ}<\delta<2.5^{\circ}$, a region along the Celestial Equator in the Northern Galactic Cap (hereafter Area 2), $140^{\circ}<\alpha<240.5^{\circ},-2.5^{\circ}<\delta<2.5^{\circ}$, and a further region at higher latitudes in the Northern Galactic Cap which is better expressed in survey coordinates, $\lambda$ (longitude) and $\eta$ (latitude) with $-17^{\circ}<\lambda<53^{\circ}, 80.75^{\circ}<\eta<86.25^{\circ}$ (hereafter Area 3). The survey coordinates are great circles on the sky and are explained in detail in Stoughton et al. (2002). The three different areas are shown in Figure 1 and the area and number of galaxies in each area is given in table 1. 
As the survey is not finished, the angular selection function is complicated. An algorithm to quantify the completeness, i.e. the fraction of galaxies that have been observed in any given region, has been developed and is described in Tegmark, Hamilton, \& Xu (2001). The completeness for any given $(\alpha, \delta)$ coordinate is returned. The completeness within the regions that have been observed is typically $>90 \%$.

We construct a volume-limited sample from the SDSS. This choice reduces the number of galaxies available, but it has several important advantages: the radial selection function is approximately uniform, thus the only variation in the space density of galaxies with radial distance is due to clustering. Therefore, no weighting scheme is required when constructing the density field; thus, the analysis is more straight forward. In addition to having nearly uniform selection function, volume-limited samples have a constant mix of galaxy types with redshift. The importance of this uniformity is demonstrated in this paper; we find that the density fields traced by the reddest and bluest galaxies do indeed exhibit different topological characteristics.

We form a volume-limited sample that extends to $z_{\max }=0.088$. This limit includes the maximum number of galaxies in the sample. In Figure 3 we plot the number of galaxies in a volumelimited sample as a function of redshift. The k-corrections we use are described in Blanton et al. (2002). The redshift limit of $z_{\max }=0.088$ corresponds to a limiting absolute magnitude of $M_{\text {lim }}=-19.86+5 \log h$ (hereafter we drop the $5 \log h$ term when discussing absolute magnitudes) in the $r^{*}$ band, assuming an average value for the k-correction. Following our previous paper (HVG02) and to maintain consistency with the Hubble Volume simulation, which we describe below in Section 2.2, we adopt a $\Omega_{\mathrm{m}}=0.3, \Omega_{\Lambda}=0.7$ cosmology when converting redshift into comoving distances. For this cosmology, the redshift limit of $z_{\max }=0.088$ corresponds to a comoving distance of $260 h^{-1} \mathrm{Mpc}$. There are 15,230 galaxies in the volume-limited sample to this distance. However, some of these survey data do not lie in any of the three areas that we consider and we only include galaxies at distances larger than $100 h^{-1} \mathrm{Mpc}$ because the wedge-shaped three-dimensional survey volume is very thin out to this distance. The final volume-limited sample contains 11,884 galaxies.

\subsection{The Simulation}

To test the robustness of our statistical methods, to estimate uncertainties in our measurement, and to test the currently best fitting variant of CDM, we use mock surveys drawn from the Virgo Consortium's Hubble Volume $z=0 \Lambda \mathrm{CDM}$ simulation. For more details on Virgo Consortium Simulations see Frenk et al. (2000) and Evrard et al. (2002). This particular simulation contains 1 billion mass particles in a cube with side $3,000 h^{-1} \mathrm{Mpc}$. The cosmological parameters of the simulation are $\Omega_{\mathrm{b}}=0.04, \Omega_{\mathrm{CDM}}=0.26, \Omega_{\Lambda}=0.7, h=0.7, \Gamma=0.17$, close to the values suggested by median statistics analysis (Gott et al. 2001). This simulation does not include any hydrodynamics

or other detailed physics but we smooth on $5 h^{-1} \mathrm{Mpc}$ scales. On this and larger scales, the dark matter fluctuations might be expected to trace the clustering pattern of galaxies. The clustering 
amplitude of the dark matter particles closely matches that of present day, optically selected galaxies so no biasing has been applied. In any case, estimation of genus as a function of area fraction reduces the sensitivity of the topology to galaxy bias, since it requires only a monotonic relation of the smoothed mass and galaxy density fields to get the same genus curve, not equality of the fields or a linear relation between them.

We construct samples that have the same geometry as the current SDSS data. We sparse sample the dark matter particles to match the number density of galaxies in the SDSS.

\section{The Genus Statistic}

The genus statistic is a quantitative measure of the topology of regions bounded by isodensity surfaces. In two-dimensions, those surfaces are simply the set of curves that separate high and low density areas. Following Melott et al. (1989), we define the 2-D genus to be

$G_{2}=$ number of isolated high density regions - number of isolated low density regions.

The genus of a contour in a two-dimensional density distribution can also be calculated using the Gauss-Bonnet theorem, in the two dimensional form, using

$$
G_{2}=\frac{1}{2 \pi} \int C d S
$$

where the line integral follows the contour and $C=r^{-1}$ is the inverse curvature of the line enclosing a high or low density region and $G_{2}$ is proportional to the Euler-Poincaré characteristic of the curve. This curvature can be positive or negative depending on whether a high or low density region is enclosed. The genus of a contour enclosing an isolated high density region is positive, whereas the genus of a contour enclosing a low density region is negative. This expression allows contributions from contours around regions that do not lie fully within the boundaries of the survey.

For a Gaussian random field, the genus has a simple analytic form. The genus per unit area is expressed as

$$
g_{2}=\frac{G_{2}}{\text { Area }}=A \nu e^{-\nu^{2} / 2},
$$

where $\nu$ is the threshold value, above which a fraction, $f$, of the area has a higher density

$$
f=(2 \pi)^{-1 / 2} \int_{\nu}^{\infty} e^{-x^{2} / 2} d x
$$

For example, at $\nu=1$ we evaluate the topology of a contour that encloses $16 \%$ of the area with highest density and at $\nu=0$, the contour is drawn at the median density. The value of the amplitude, $A$, depends on the shape of the smoothed power spectrum,

$$
A=\frac{<k^{2}>}{2(2 \pi)^{3 / 2}}
$$


and $<k^{2}>$ is the square of the wavenumber, $k$, averaged over the two-dimensional power spectrum.

The steps to calculating the genus are as follows:

- Construct a random catalog of points for each SDSS area that has the same angular and radial selection function as the galaxies but with much higher number density.

- Bin the galaxies (each area is considered separately) and the random points on a twodimensional grid. We have $256^{2}$ cells in each grid. Calculate the density of data and random points in each cell.

- Smooth the data and random density field with a Gaussian kernel

- Divide the smoothed data density field by the smoothed random density field.

- Mark the cells that lie outside the survey (i.e., the cells with density=0 in the unsmoothed random density field) with a negative value. These cells are then ignored by CONTOUR2D.

- Run CONTOUR2D to compute the genus at several density thresholds.

CONTOUR2D (Melott 1989) ${ }^{1}$ uses a two-dimensional variant of the angle deficit algorithm described by Gott et al. (1986). The density contour is specified by the fractional area contained in the high density region of the smoothed density field. The program classifies all cells as high or low density then evaluates the genus of the contour surface by summing the angle deficits at all vertices that lie on the boundary between the high and low density regions.

\subsection{The Smoothing Length}

We smooth the data and random point density distributions by convolving the density field with a Gaussian of the form $\exp \left(-r^{2} / 2 \lambda^{2}\right)$, where $\lambda$ is the smoothing scale. Note that this definition of the Gaussian smoothing length $\lambda$ differs from some earlier papers on the genus statistic (some earlier papers use $\left.\exp \left[-r^{2} / \lambda^{2}\right]\right)$.

Vogeley et al. (1994) describe a series of desiderata that the smoothing length should satisfy. First, the smoothing length should be larger than the grid cell. For smoothing, we bin the galaxies in each of the three SDSS regions onto a $256^{2}$ grid with side length $575 h^{-1} \mathrm{Mpc}$, thus the grid cell size is of order $2 h^{-1} \mathrm{Mpc}$.

The second criterion is that we require the variance due to clustering, $\sigma_{\lambda}$, on the smoothing scale, $\lambda$, be larger than the Poisson error on this same scale. This ensures that we are dominated

\footnotetext{
${ }^{1}$ Note that the version of CONTOUR2D published in Melott (1989) contains a sign error in line 19 of the OUT subroutine.
} 
by clustering signal rather than shot noise. For top-hat smoothing with radius $\lambda$ (the results for a Gaussian smoothing are of the same order), the constraint would be

$$
\begin{gathered}
\frac{\delta N_{\text {clustering }}}{N}>\frac{\delta N_{\text {poisson }}}{N} \\
\sigma_{\lambda}>\frac{1}{\sqrt{N}}=\frac{1}{\lambda \sqrt{\pi \bar{\mu}}} \\
\lambda>\left(\sigma_{\lambda} \sqrt{\pi \bar{\mu}}\right)^{-1}
\end{gathered}
$$

where $\bar{\mu}=N /$ A, the number of galaxies in the sample divided by the area of the sample. For a smoothing scale of $5 h^{-1} \mathrm{Mpc}$ this value is small, of order $0.5 h^{-1} \mathrm{Mpc}$.

The third criteria is that we want the smoothing length to be larger than the average intergalaxy separation. This avoids, for example, erroneously treating a single galaxy as a "cluster." This is satisfied if

$$
\lambda>(\bar{\mu})^{-1 / 2} .
$$

For the three SDSS regions, the value of $\bar{\mu} \sim 0.1$, therefore $\lambda$ is required to be larger than $3 h^{-1} \mathrm{Mpc}$. At the inner edge of the Survey, the wedge shape is thinner and hence the value of $\mu$ is less than the average value. At the inner edge of the wedge, the value of $\mu^{-1 / 2} \sim 4.5 h^{-1} \mathrm{Mpc}$.

The number of resolution elements in the survey depends on the smoothing length applied. We wish to make this number as large as possible. The number of resolution elements, $N_{\text {res }}$, in two-dimensions is roughly

$$
N_{\text {res }}=\frac{A_{\text {sample }}}{\pi \lambda^{2}}
$$

therefore it is advantageous to use the smallest value of $\lambda$ consistent with the shot noise constraints possible. We choose a smoothing length of $5 h^{-1} \mathrm{Mpc}$ for most of our analysis. Table 1 lists the number of resolution elements for each area. Using a $5 h^{-1} \mathrm{Mpc}$ smoothing length we have at least

400 resolution elements in the measurement of the genus from each area. For comparison, the 2dFGRS extends to greater depth so even with a smoothing length of $10 h^{-1} \mathrm{Mpc}$, HVG02 used of order 400 resolution elements in each of the NGP and SGP slices.

\subsection{Errors}

We use the simulations as a method for estimating the errors on the genus curve measured from the SDSS. The two-point clustering amplitude of the $\Lambda$ CDM Hubble Volume simulation is close to that of present day, optically selected galaxies (see Hoyle et al. 1999).

In HVG02, we investigated calculating the full covariance matrix and employing that matrix when $\chi^{2}$ fitting the data to a Gaussian random curve. We found that 20 mock catalogs were not sufficient to adequately describe the full covariance matrix, even if the higher order clustering was truly described by the $\Lambda \mathrm{CDM}$ simulation. Therefore we use the 20 mock catalogs only to estimate 


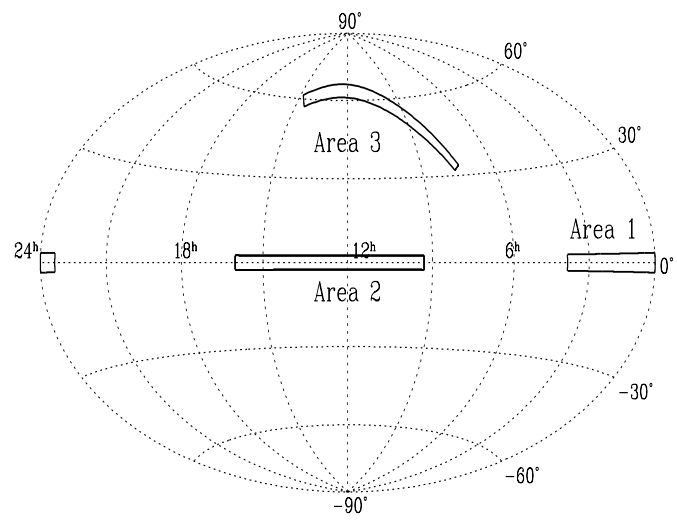

Fig. 1.- An Aitoff projection in celestial coordinates of the approximate areas covered by the three SDSS regions from which we measure the two-dimensional genus. Coordinate limits for these regions are described in the text.

\begin{tabular}{cccccccccc} 
Data & Area & $N_{\text {gal }}$ & $N_{\text {res }}$ & Amp & Rank & Significance & Shift & Rank & Significance \\
\hline Area 1 & 35,000 & 2,343 & 435 & 8.6 & 13 & $0.62<1 \sigma$ & 0.05 & 14 & $0.66<1 \sigma$ \\
Area 2 & 54,000 & 6,970 & 685 & 14.6 & 4 & $0.19 \sim 1 \sigma$ & -0.15 & 3 & $0.14 \sim 1 \sigma$ \\
Area 3 & 35,000 & 2,571 & 435 & 7.4 & 3 & $0.14 \sim 1 \sigma$ & 0.2 & 17 & $0.81 \sim 1 \sigma$ \\
\hline Combined & 124,000 & 11,884 & 1555 & 28.1 & 4 & $0.19 \sim 1 \sigma$ & -0.05 & 8 & $0.38<1 \sigma$ \\
\hline
\end{tabular}

Table 1: Properties and rank of the amplitude and shift for the genus curves from the three areas and the summed genus curve as compared to the 20 realizations from the simulation. The area is given in units of $h^{-2} \mathrm{Mpc}^{2}$ and the number of resolution elements is calculated assuming a smoothing length $5 h^{-1} \mathrm{Mpc}$. The samples are volume-limited with $z_{\max }=0.088$ corresponding to a magnitude limit of $\mathrm{M}_{\lim }=-19.86$ in the $\mathrm{r}^{*}$ band. The rank is that of each dataset with respect to the 20 mock catalogs, i.e. a ranking of 9 means there are 8 mock catalogs with smaller values and 12 with larger values of the feature we are testing. 


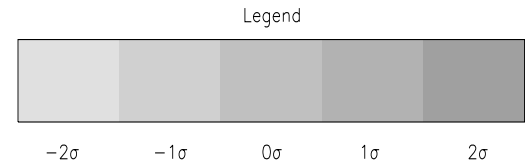

Smoothed Area $1 \quad$ Smoothed Area $2 \quad$ Smoothed Area 3
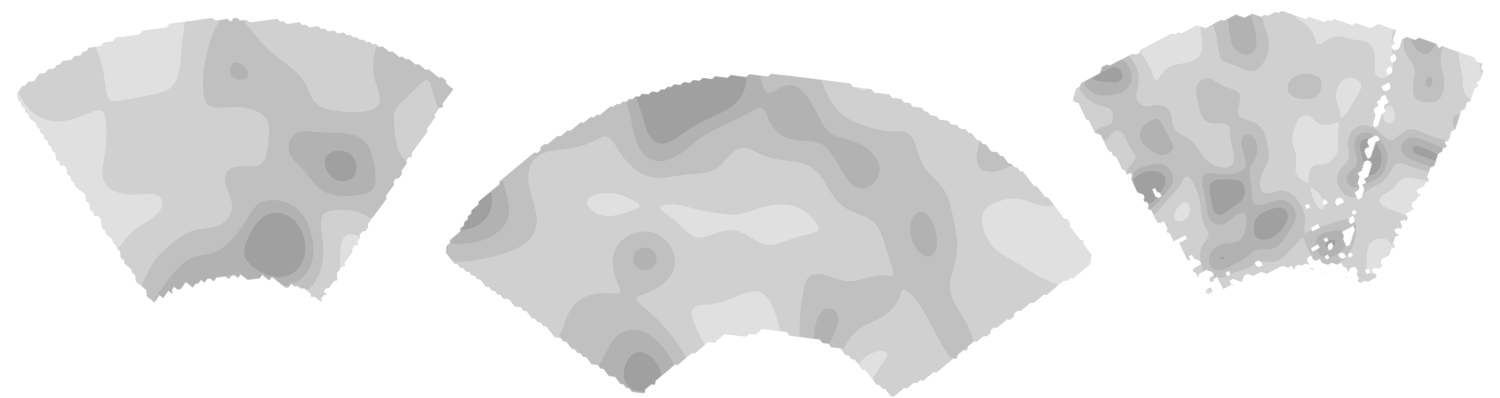

Unsmoothed Area 1

Unsmoothed Area 2

Unsmoothed Area 3
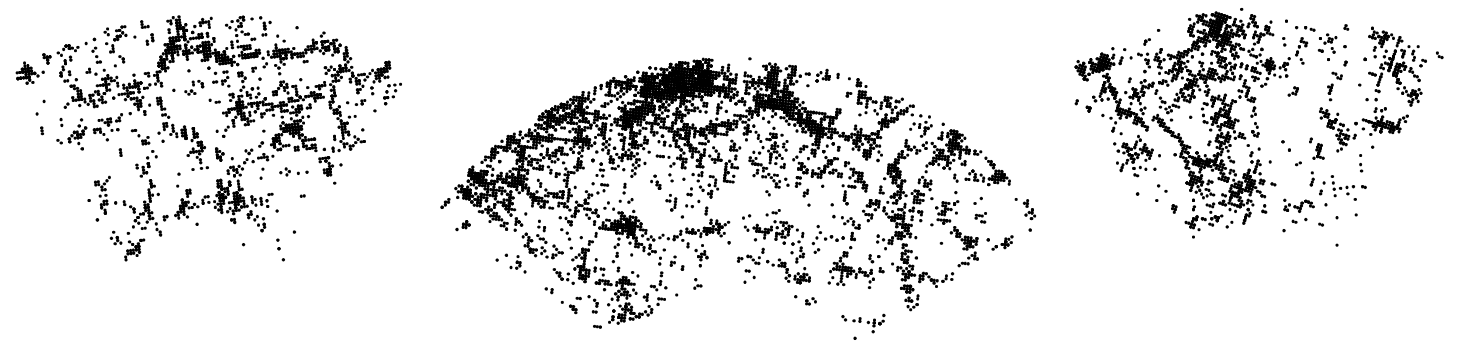

Fig. 2.- Smoothed and unsmoothed maps of the density of objects in the SDSS slices. Darker contours correspond to denser regions. In the smoothed plots, the darkest contours are $2 \sigma$ overdense regions and the lightest contours are the $2 \sigma$ under-dense regions. The $2 \mathrm{D}$ smoothing kernel is a Gaussian with dispersion $\lambda=5 h^{-1} \mathrm{Mpc}$. Small differences in the qualitative appearance of the smoothed and unsmoothed maps are due to incompleteness of the survey. This incompleteness and the varying thickness of the slices with redshifts are accounted for in the smoothed maps. In Area 3 a region of incomplete spectroscopy causes the narrow underdensity which is excluded from our analysis. 
the variance of each genus measurement. As the amplitude of the SDSS genus curve closely matches that of the simulation, it seems reasonable to use the errors from the simulation as estimates for the errors in the data.

In the figures below we plot estimated uncertainties for every other point in the genus curve. This choice reflects the strong covariance of genus estimates and makes the plots easier to read.

\subsection{Combining the Genus Curves}

To combine estimates of the genus from different samples, whether from sub-regions of the same survey, or from different surveys, one should not simply add the genus curves $G(\nu)$. A better procedure is to compute $G(\rho)$ for each sample, then combine the results into a summary statistic $G(\nu)$, using the relationship $\nu(\rho)$ derived from the combined sample. This procedure, detailed in this section below, avoids errors caused by fluctuations in the mean density and the density probability distribution.

CONTOUR2D computes the genus at a set of specified area fractions for the low-density region enclosed by the isodensity contour. This fraction is parametrized by $\nu$, as in eq. (4). The code iterates to find the density threshold corresponding to this area fraction, draws the contour, computes the genus of this contour and returns $\rho$ and $G$ for each area fraction. The value of the density threshold $\rho(\nu)$ corresponding to fixed $\nu$ can vary among different samples under consideration due to variations in large-scale structures present in the samples. Thus, rather than naively summing genus curves for different samples at each value of $\nu$, we sum the values of the genus at matching density thresholds $\rho$ when calculating the total genus curve of the survey.

The procedure is as follows: First the area fraction and the genus is found for each area as a function of $\rho$. We compute the genus for a large number of threshold values, so that the curves may be interpolated to later find $G(\rho)$ at any value of $\rho$. We then calculate the average area fraction at each $\rho$ value by summing the three individual area fractions, weighted by the areas of each region. The total genus at each density value is found by summing the three genus values as the total genus takes into account the different areas. This yields the total genus of the SDSS as a function of the area fraction. The final step is to convert the area fraction back into $\nu$ values. The resulting curve

is identical to the curve that would be obtained by binning the data for all of the sub-regions onto a larger grid and simultaneously computing the genus for all three regions.

Note carefully that, at the negative $\nu$ end of the genus curve, changes in the area fraction lead to very small changes in the density threshold (i.e., the gradient of the density field is quite shallow). As a result, in some cases the genus curves of the combined sample are neither computed nor plotted at very low values of $\nu$. In contrast, at the high $\nu$ end, changes in the area fraction can correspond to quite large changes in the density threshold (due to the steeper density gradient), allowing the high $\nu$ end of the genus curve to be better sampled if we binned linearly in density. 


\section{Results}

\subsection{Genus of Volume Limited Samples of the SDSS}

In Figure 4 we show the genus curves from the three SDSS areas. The data have been smoothed with a Gaussian of width $5 h^{-1} \mathrm{Mpc}$ in all cases and we show the total genus of each area. In Figure 5 we combine the genus curves of the three areas, as described in Section 3.3, to present the total genus for the sample of SDSS galaxies presented here. We show the results for two values of the smoothing length, $5 h^{-1} \mathrm{Mpc}$ (left) and $10 h^{-1} \mathrm{Mpc}$ (right). For comparison, we plot the best fit genus curve of a Gaussian random field distribution, shown by the dashed line. The genus curve found using a $10 h^{-1} \mathrm{Mpc}$ smoothing length is noisier due to the smaller number of resolution elements. We focus on the $5 h^{-1} \mathrm{Mpc}$ results for the remainder of the analysis.

As described in HVG02, we use the Mann-Whitney Rank Sum test to compare the data to the mock catalogs. Table 1 shows the comparison with 20 mock catalogs. We measure the amplitude of the genus curve of the data and mock catalogs by $\chi^{2}$ fitting to a Gaussian curve (equation 3 ) with varying amplitude. The shift of the curve is found simply by measuring the zero-crossing of the genus curve.

We find that the amplitude of the genus of the SDSS lies slightly below the median genus amplitude of the simulated catalogs (rank 11). This decrement appears to be because the genus curve of the SDSS peaks at smaller absolute $\nu$ values than the Gaussian random curve and the simulation, as shown in Figures 5 and 6, respectively. For the SDSS, the width $\left|\nu_{\max }-\nu_{\min }\right| \sim 1.6$, whereas for a Gaussian curve $\left|\nu_{\max }-\nu_{\min }\right|=2$ and the genus measured from the simulation, which assumes Gaussian initial conditions, also has $\left|\nu_{\max }-\nu_{\min }\right| \sim 2$. We do not see this effect in the 2dFGRS data. In fact, the 2dFGRS data exhibit a slight broadening in the peak-trough separation. Neither do we see any narrowing of the SDSS genus curve when we use a $10 h^{-1} \mathrm{Mpc}$ smoothing length, although the smaller number of resolution elements causes the genus curve to be noisier for $10 h^{-1} \mathrm{Mpc}$ smoothing and locating the peaks is difficult. This narrowing in the peak-trough separation in the SDSS data using the $5 h^{-1} \mathrm{Mpc}$ smoothing length is in the opposite sense to the results of Vogeley et al. (1994), who found that the three-dimensional genus curve of the CfA survey was slightly broader at the $85 \%$ level for small choices of the smoothing length $\left(\lambda<12 h^{-1} \mathrm{Mpc}\right)$.

The zero-point shifts of the data are more consistent with the simulation. Area 2 has a slight negative shift but on average the zero crossing point agrees with the simulation. The fact that it lies at less than zero suggests there is a slight excess of clusters (a meatball shift) in both the data and the simulation, as seen previously in the 2dFGRS (HVG02).

We also compare the SDSS genus curve to the 2dFGRS genus curve. The total area of the 2dFGRS sample analyzed in HVG02 is approximately double that of the SDSS slices analyzed here so the 2dFGRS curve has been scaled in Figure 6 to match the SDSS curve. Here we plot a revised genus curve for the $2 \mathrm{dFGRS}$ that uses the more accurate procedure described above in section 3.3 for combining the genus curves of the North and South regions of that survey. The genus curves for 


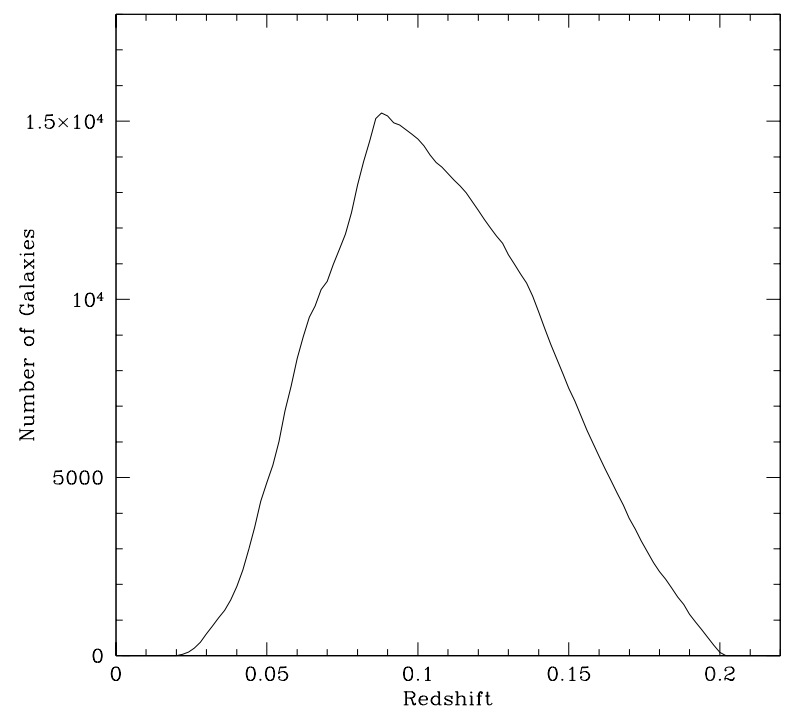

Fig. 3. - Number of galaxies in a volume-limited sample, selected from all the SDSS galaxies, as a function of the redshift limit. The sample size peaks at redshift $z=0.088$, which corresponds to an absolute magnitude limit of $M_{\lim }=-19.86$ in the $r^{*}$ band.
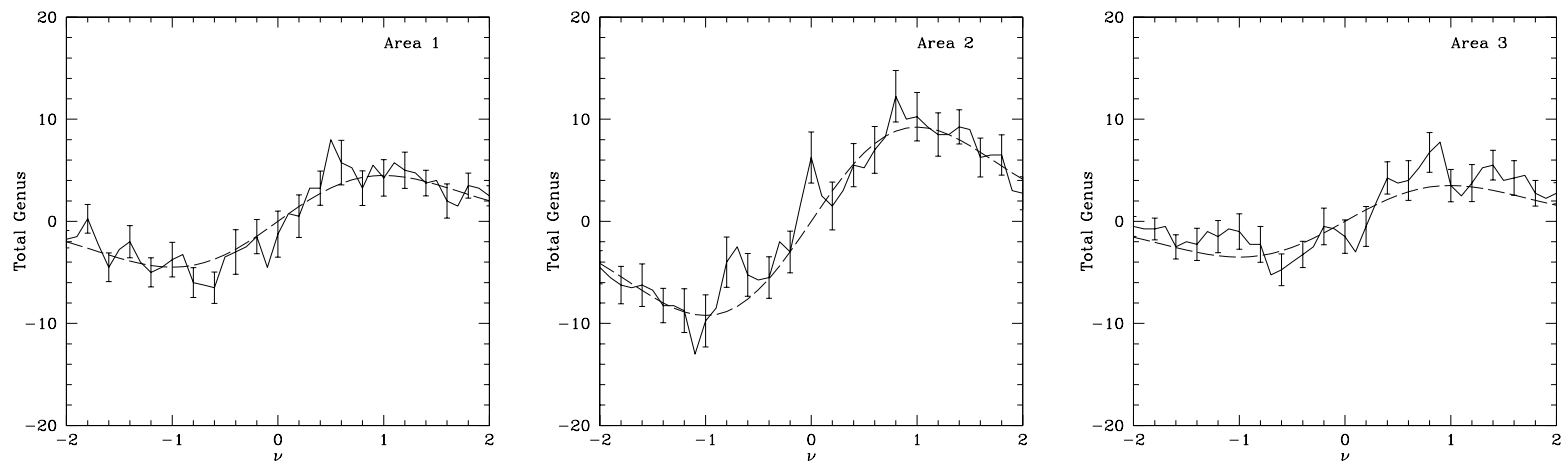

Fig. 4.- Genus curves of the three SDSS slices. A $5 h^{-1} \mathrm{Mpc}$ smoothing length is used. Errors are estimated from 20 mock catalogs drawn from the Hubble Volume $\Lambda$ CDM simulation. Dashed curves show the best fit (using $\chi^{2}$ ) Gaussian random field genus (equation 3). 

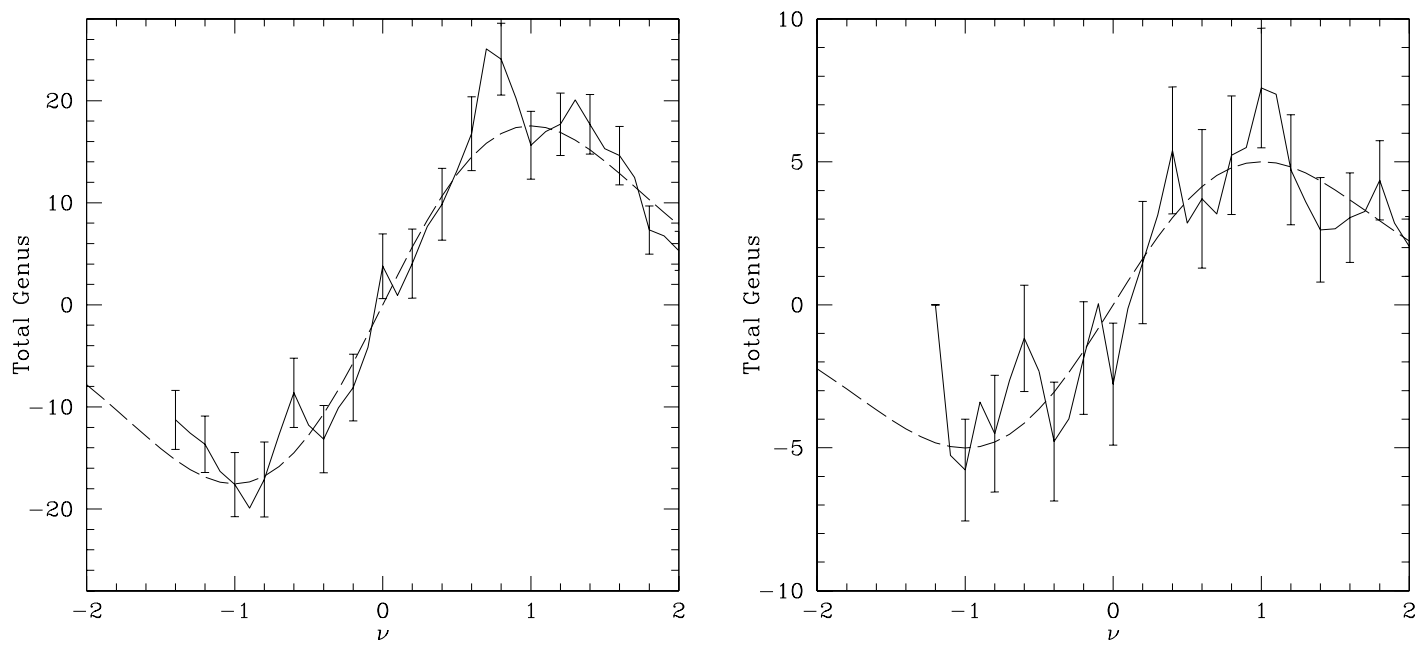

Fig. 5.- The total genus curve (combined as described in Section 3.3) of the SDSS calculated using a $5 h^{-1} \mathrm{Mpc}$ smoothing length (left hand plot) and a $10 h^{-1} \mathrm{Mpc}$ smoothing length (right hand plot). Dashed curves show the best fit (using $\chi^{2}$ ) Gaussian random field genus (equation 3). The combined genus is not computed for the lowest values of $\nu$, as described in Section 3.3.
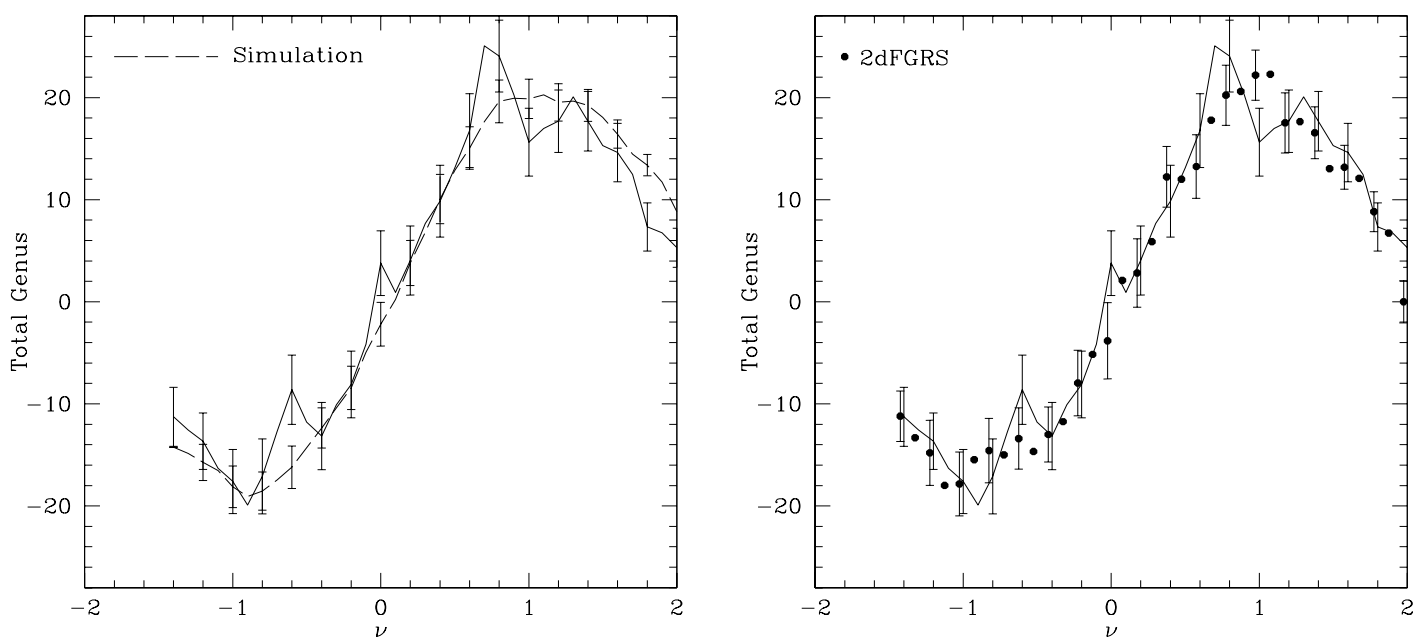

Fig. 6. - The total genus curve of the SDSS compared to the average genus curve of the 20 mock catalogs, drawn from the Hubble Volume $\Lambda$ CDM simulation, described in Section 2.2 (left plot), and the 2dFGRS curve similar to that given in HVG02 but recombined as described in this paper (right hand plot). Note that the 2dFGRS points are slightly offset to the left for clarity, and that every other 2dFGRS point has an error bar attached. The points without error bars have similar errors. A $5 h^{-1} \mathrm{Mpc}$ smoothing length is used. 
the full SDSS and 2dFGRS samples agree within the estimated uncertainties. However the cluster excess in the 2dFRGS genus curve found in HVG02 is less apparent when we compute the genus curve for the full sample using our new more accurate method.

\subsection{Genus as a Function of Galaxy Type}

A unique feature of the SDSS project is that medium-resolution spectroscopy and five-band CCD photometry are obtained for all the objects in the galaxy redshift survey. Details of these measurements may be found in Stoughton et al. (2002).

Here we examine whether the genus curve depends on the color of the galaxy. Red, elliptical galaxies are believed to be preferentially found in high density regions whereas blue spiral galaxies may be found preferentially in lower density regions. This difference may lead to quantitatively different genus curves, as discussed in Gott, Cen, \& Ostriker (1996), where it was predicted that elliptical galaxies may show a shift towards a meatball topology relative to spiral galaxies. Therefore, we split the sample by $u^{*}-r^{*}$ color, which was found to be a good indicator of galaxy type by Strateva et al. (2001). The data divide into three equal sized samples if we split the sample at $u^{*}-r^{*}<2.35$ and $u^{*}-r *>2.86$.

We investigate whether reducing the number of galaxies in the sample has an effect on either the shape of the genus curve or on the size of the errors. By sparse sampling our mock catalogs at a rate of 1 in 3, we find that the genus curve is not changed significantly in shape, nor do the error bars increase by more than $10 \%$. It appears that the density of galaxies is high enough for the clusters and voids to be well sampled, thus the genus curve is robust to sub-sampling of the survey. In other words, the uncertainties on the genus curve are caused by different structures within the samples, not by Poisson fluctuations in the galaxy density field.

In the SDSS samples, we find (see Figure 7) noticeable differences between the genus curves of the reddest and bluest galaxies. The red galaxies (filled circles) appear to have a meatball shift as compared to the bluest galaxies (open circles) and the full sample. In the two dimensional genus, a meatball shift occurs when the genus remains positive - dominated by isolated clusters - at negative values of $\nu$, i.e., below the median density. This behavior for the reddest galaxies is consistent with the prediction of Gott et al. (1996), reflecting the higher concentration of early-type galaxies in the highest density regions. None of the one-in-three sampled mock catalogs from the Hubble Volume simulation exhibit a meatball shift of this magnitude.

While the reddest third of the galaxy sample exhibits a shift reflective of an excess of isolated clusters, this result only became clear after combining the three sub-regions as described in section 3.3. This effect was not clearly apparent in the genus curves of the sub-regions. In other words, the sample we examine is just large enough to reveal this effect, which suggests that this might be a statistical fluke. We will repeat this analysis with a larger sample as it becomes available. The amplitude of the genus curves also appear to be suppressed when we split the samples by color. 


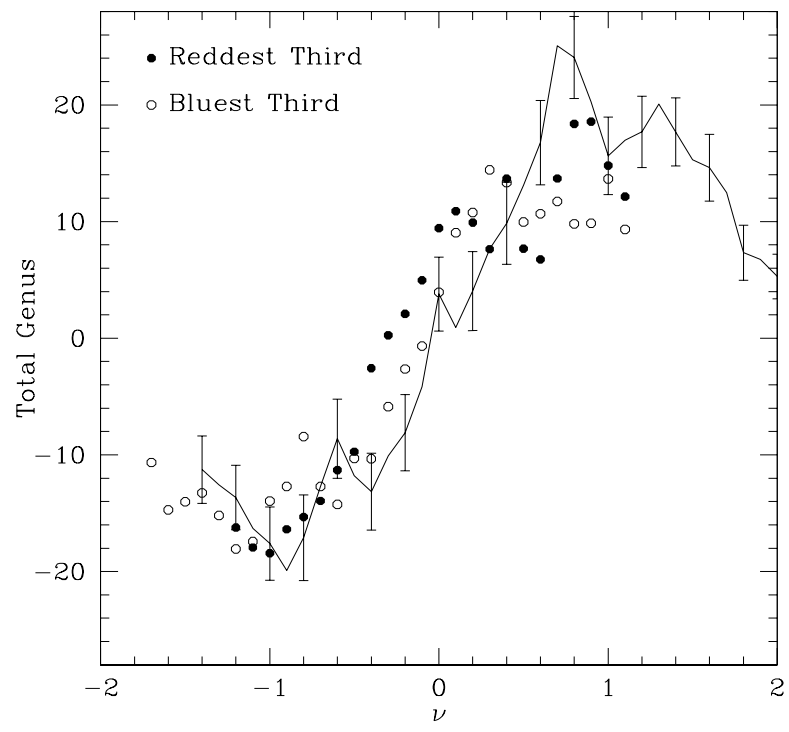

Fig. 7.- Genus curve of the reddest third of the sample (filled circles) compared to the genus curve of the bluest third of the sample (open circles) selected in $u^{*}-r^{*}$. The solid line shows the genus curve of the full sample. For clarity, we only plot the errors for the full sample. Errors for the red and blue samples are similar in size. The genus curve of the reddest third shows a meatball shift (toward $\nu<0$ ) relative to the bluest third and the full sample. 
The cause of the latter effect is not clear.

It is important to emphasize that this measurement reveals a difference in the topology of the distribution of galaxies segregated by color, rather than simply reflecting the well-known morphology-density relation (Dressler 1980; Postman \& Geller 1984). By comparing genus curves as a function of area fraction (see eq. 4), differences in the density probability distribution have already been taken into account.

This observed dependence on galaxy color of the genus of the smoothed galaxy density field suggests that there is not a simple one-to-one mapping between the mass and galaxy density. Note that our use of the volume fraction as the independent variable in the genus analysis is partly motivated by the hope that smoothing on a sufficient scale yields a monotonic relationship between mass density in the initial conditions and galaxy density at the observed epoch. Departure from monotonicity in the mass-galaxy relationship, non-locality of this relationship, or stochasticity in this mapping could violate this assumption, if the smoothing scale is smaller than the scale of such effects. Thus, dependence on galaxy color and the departures from the Gaussian prediction of the full sample may indicate significant complexity in the mass-galaxy relationship on smoothing scales $\lambda \sim 5 h^{-1} \mathrm{Mpc}$.

\section{Conclusions}

We estimate the two-dimensional genus of an early sample of SDSS galaxies, which is one of the two largest samples analyzed for $2 \mathrm{D}$ topology. We find that the genus is consistent with that of a $\Lambda$ CDM simulation and has an approximately Gaussian shape, although there is a slight meatball shift, consistent with the simulation. The peaks of the genus curve appear at slightly smaller absolute $\nu$ values than would be found for a Gaussian distribution or for the $\Lambda$ CDM simulation, but the statistical significance of this effect is marginal.

We compare the SDSS results with the 2dFGRS genus curve and find that the two curves have approximately the same shape, when renormalized due to the different areas of the surveys.

To test for segregation of galaxies by type, we also split the SDSS data into thirds using a color criteria and measure the genus curves of these samples. We find that the genus curve of the reddest galaxies exhibits a meatball shift, as predicted by N-body/hydrodynamical simulations (Gott et al. 1996). However, this effect may sensitively depend on the number of high-density regions (clusters) in the sample. The current sample is still relatively small (only 10-20 massive clusters lie within a typical volume of this size), thus examination of larger samples is necessary to confirm this finding. The completed SDSS galaxy redshift survey will include roughly 10 times more galaxies, allowing us to further test the detailed dependence of topology on galaxy properties. 


\section{Acknowledgments}

Funding for the creation and distribution of the SDSS Archive has been provided by the Alfred P. Sloan Foundation, the Participating Institutions, the National Aeronautics and Space Administration, the National Science Foundation, the U.S. Department of Energy, the Japanese Monbukagakusho, and the Max Planck Society. The SDSS Web site is http://www.sdss.org/.

The SDSS is managed by the Astrophysical Research Consortium (ARC) for the Participating Institutions. The Participating Institutions are The University of Chicago, Fermilab, the Institute for Advanced Study, the Japan Participation Group, The Johns Hopkins University, Los Alamos National Laboratory, the Max-Planck-Institute for Astronomy (MPIA), the Max-Planck-Institute for Astrophysics (MPA), New Mexico State University, Princeton University, the United States Naval Observatory, and the University of Washington.

MSV acknowledges support from NSF grant AST-0071201 and a grant from the John Templeton Foundation. JRG acknowledges the support from NSF grant AST-9900772. We thank Ravi

Sheth and Chris Miller for useful conversations. We thank the anonymous referee for insightful comments.

\section{REFERENCES}

Bardeen, J. M., Steinhardt, P. J., \& Turner, M. S., 1983, Phys. Rev., 28, 679

Blanton, M. R., et al. 2001, astro-ph/0105535

Blanton, M. R., et al. 2002, AJ

Canavezes, A., et al., 1998, MNRAS, 297, 777

Coles, P., \& Plionis, M., 1991, MNRAS, 250, 75

Colley, W. N., 1997, ApJ, 489, 471

Connolly, A. J., et al. 2002, ApJ submitted, astro-ph/0107417

Dodelson, S., et al. 2002, ApJ submitted, astro-ph/0107421

Dressler, A. 1980, ApJ, 236, 351

Eisenstein, D. J. et al. 2001, AJ, 122, 2267

Evrard, A. E. et al., ApJ accepted, astro-ph/0110246

Frenk, C.S.. et al. 2000, astro-ph/0007362

Fukugita, M., Ichikawa, T. , Gunn, J. E. , Doi, M. , Shimasaku, K. \& Schneider, D. P., 1996, AJ, 111,1748 
Gott, J. R. III, et al. 1989, ApJ, 340, 625

Gott, J.R. III, Cen, R. \& Ostriker, J. P., 1996, ApJ, 465, 499

Gott, J. R. III, Melott, A. L., \& Dickinson, M., 1986, ApJ, 306, 341

Gott, J.R. III, Vogeley, M.S., Podariu, S., \& Ratra, B. 2001, ApJ, 549, 1

Gunn, J., 1998, ApJ, 116, 3040

Hogg, D.W., Finkbeiner, D.P., Schlegel, D.J., \& Gunn, J.E. 2001, AJ, 122, 2129

Hoyle, F., Baugh, C. M., Shanks, T. \& Ratcliffe, A., 1999, MNRAS, 309, 659

Hoyle, F., Vogeley, M.S. \& Gott, J. R. III, 2002, ApJ, 570, 44

Infante, L. et al. 2002, ApJ, 567, 1551

Lupton, R. H., 2002, in preparation

Melott, A. L., 1987, Topology of the Universe: Motivation for the Study of Large -Scale Structure, in Proceedings of the XIIIth Texas Symposium on Relativistic Astrophysics, M. Elmer ed, 1987, World Scientific Publishing

Melott, A. L., 1989, Physics Reports, 193, 1

Melott, A. L., Cohen, A. P., Hamilton, A., Gott, J. R. III, \& Weinberg, D., 1989, ApJ, 345, 618

Moore, B. et al. 1992, MNRAS, 256, 477

Park, C., Gott, J. R. III, da Costa, L. N., 1992, ApJ, 392, L51

Park, C., Gott, J. R. III, Melott, A. L., \& Karachentsev, I. D., 1992, ApJ, 387, 1

Pier, J., et al. 2002, AJ submitted

Plionis, M., Valdarnini, R. \& Coles, P., 1992, MNRAS, 258, 114

Postman, M., \& Geller, M.J. 1984, ApJ, 281, 95

Protogeros, Z. A. M. \& Weinberg, D. H. 1997, ApJ, 489, 457.

Scranton, R. et al. 2002, ApJ submitted, astro-ph/0107416

Smith, J.A., et al. 2002, AJ, 123, 2121

Springel, V. et al. 1998, MNRAS, 298, 1169

Strateva, I. et al., 2001, AJ, 122, 1861 
Strauss, M., et al. 2002, AJ submitted

Stoughton, C. et al. 2002, AJ, 123, 485

Szalay, A. S., et al. 2002, ApJ submitted, astro-ph/0107419

Szapudi, I., et al. 2002, ApJ, 570, 75

Tegmark, M., Hamilton, A. J. S., \& Xu, Y., 2001, MNRAS submitted, astro-ph/0111575

Tegmark, M., et al. 2002, ApJ, 571, 191

Vogeley, M.S., Park, C., Geller, M. J., Huchra, J.P., \& Gott, J.R. III, 1994, 420, 545

York, D. G., et al. 2000, AJ, 120, 1579

Zehavi, I., et al. 2002, ApJ, 571, 172 